\title{
Multi-walled carbon nanotubes conjugated to tumor protein enhance the uptake of tumor antigens by human dendritic cells in vitro
}

Cell Research (2010) 20:1170-1173. doi:10.1038/cr.2010.133; published online 14 Sepember 2010

\section{Dear Editor,}

Anti-tumor immunotherapy is an important form of adjuvant cancer treatment $[1,2]$. While chemotherapy encounters the obstacles of drug toxicity and resistance, immunotherapy usually has limited adverse effects, good patient tolerance, and the potential to significantly improve the prognosis [1-4]. Some clinical trials of immunotherapy generated promising results in treating malignancies such as malignant melanoma, glioblastoma multiforme, or renal cell carcinoma, which tend to respond poorly to chemotherapies [3-5]. Dendritic cells (DC) play a crucial role in generating specific immune reaction to antigens, which generally need to be ingested, processed and presented by DC, before triggering B cell- or $\mathrm{T}$ cell- mediated responses. This core immune mechanism has been utilized in designing DC-based anticancer immunotherapy, whereby a patient's DC are expanded with in vitro culture, stimulated with tumor antigen, and infused back to the body to elicit anticancer reactions [6]. DC-based therapy is an important type of anticancer immunotherapy and has generated promising results in treating difficult malignancies such as glioblastoma or malignant melanoma in clinical trials $[4,6]$. The therapy was also demonstrated effective in treating hormone refractory prostate cancer [6]. As the clinical trials exploring the efficacy of DC-based therapy are underway, there are still problems to solve, including the development of more efficient methods for loading DC with tumor antigens so as to further overcome the immune escape mechanism of tumor and benefit more patients. An important issue of DC-based anticancer immunotherapy, therefore, is to develop new technologies that can further enhance the efficacy for DC stimulation by available tumor antigens.

Carbon nanotubes (CNT) have been shown to have potential applications in multiple biomedical fields [7], especially as transporters for intracellular delivery of various bioactive molecules such as peptides, proteins,
DNAs, RNAs, or drugs. Given the excellent features of CNT as transporters of proteins and peptides across the cell membrane, and the primary goal of DC therapy to enhance anticancer immunity by channeling more tumor antigens into DC, it will be interesting to explore whether CNT can be utilized to facilitate the transportation of tumor antigens into DC. In this study, we investigated whether the conjugates of carbon nanotubes and tumor protein (CNT-TumorP) would influence the uptake of tumor antigen by human DC and the capacity of DC to induce anticancer cytotoxicity in vitro.

Functionalized multi-walled carbon nanotubes (referred to as CNT hereupon) were prepared using a published protocol [8]. Briefly, an oxidation/sonication procedure was utilized to introduce carboxyl groups to the CNT for solubilization and subsequent protein conjugation (Supplementary information, Figure S1). The CNT had an average length of 500-800 $\mathrm{nm}$ and an average diameter of 20-30 $\mathrm{nm}$ (Figure 1A). MCF7 human cancer cells were lysed and tumor lysate protein (TumorP) was extracted. The advantage of tumor lyastes as tumor antigen in immunotherapy is that they are readily obtainable, and can be applied to many solid tumors, irrespective of the genetic makeup of the tumor cells. Moreover, human clinical studies based on tumor lysates have generated promising results in some of the trials $[4,6]$. Tumor protein was labeled with fluorescein-5-isothiocyanate (TumorP-FITC), and then covalently conjugated to CNT (CNT-TumorP-FITC), in the presence of the coupling agent $N$-ethyl- $N$ '-(3dimethylaminopropyl) carbodiimide (EDAC, Figure 1B) as described by Meng et al. [8], with a loading rate of approximately $4 \mathrm{mg}$ protein per $\mathrm{mg}$ of CNT. Human DC were cultured from fresh peripheral blood of healthy donors per standard protocol employing GM-CSF and IL-4 $[4,6]$. Two groups of DC were co-incubated with TumorP-FITC and CNT-TumorP-FITC separately. After thorough wash, the uptake of FITC-labeled tumor protein by DC was evaluated by flow cytometry, which is the 
A

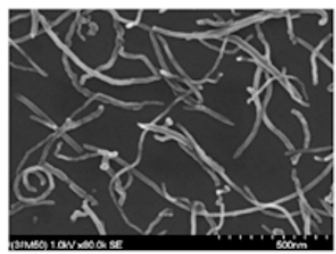

B

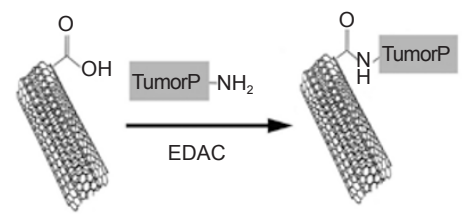

C
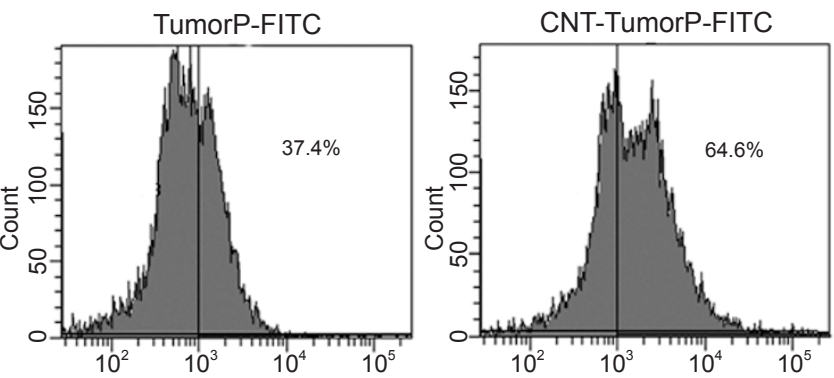

D

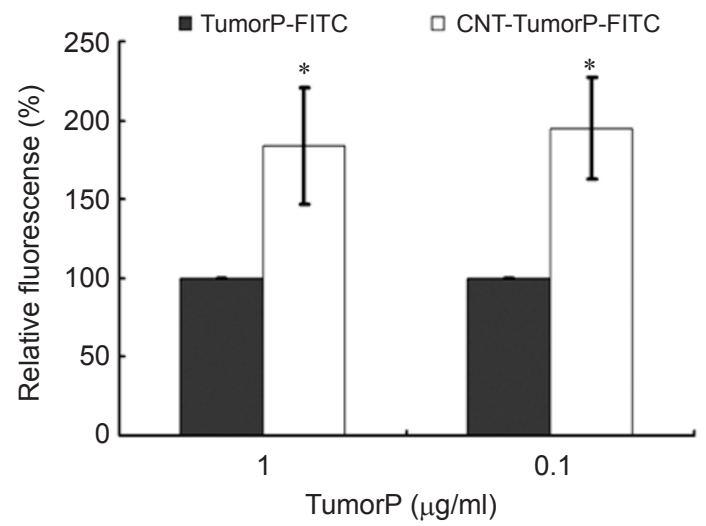

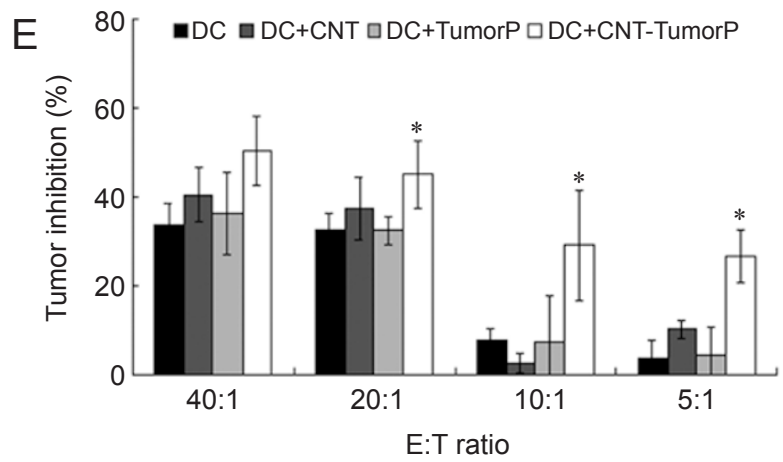

$\mathrm{F}$

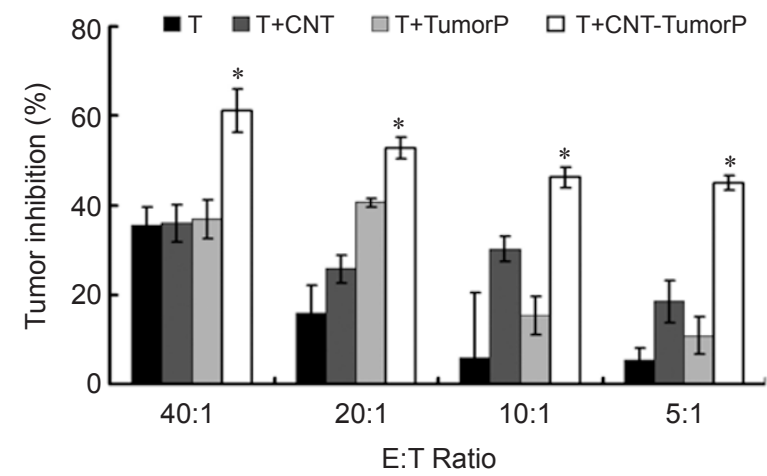

G

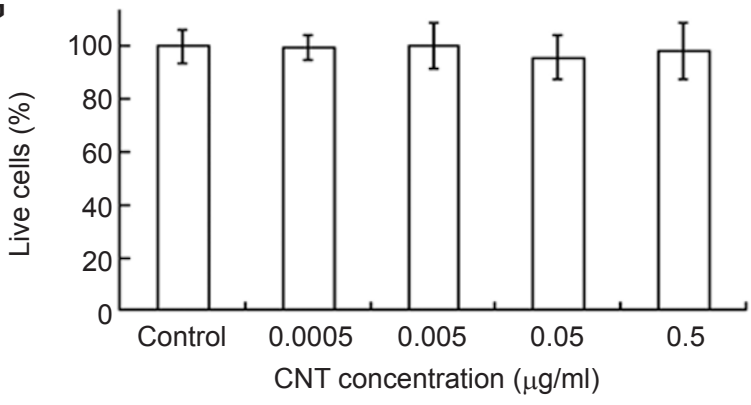

Figure 1 Effects of CNT-TumorP on processing of tumor antigens by human DC. (A) Scanning electron microscopy image of CNT. The measure bar corresponds to $500 \mathrm{~nm}$. (B) Schematic drawing of CNT and the functionalizing process with tumor lysate protein. (C) Flow cytometry histograms of DC after co-incubation with either TumorP-FITC (left) or CNT-TumorP-FITC (right). (D) Relative fluorescence intensities of DC incubated with different concentrations of TumorP or CNT-TumorP $(n=$ $6, \pm$ SD). The fluorescence intensity of DC incubated with TumorP-FITC was used as the baseline reference, with its value designated as $100 \%$. The star indicates a statistically significant difference between the TumorP-FITC and the CNT-TumorPFITC groups $(P<0.05)(E)$ Anti-tumor cytotoxicity generated by lymphocytes mixed with DC that had been exposed to either CNT, TumorP, or CNT-TumorP $(n=6, \pm \mathrm{SD})$. The star indicates a statistically significant difference between the DC+CNTTumorP and the DC+TumorP groups $(P<0.05)$. (F) Anti-tumor cytotoxicity generatated by PBMC that had been exposed to either TumorP, CNT, or CNT-TumorP. The cytotoxicity was assayed at various E:T ratios $(n=6$, \pm SD). The star indicates a statistically significant difference between the T+TumorP and the T+CNT-TumorP groups $(P<0.05)(\mathbf{G})$ Percentage of live MCF7 cells $72 \mathrm{~h}$ after co-incubation with CNT of various concentrations $(n=6, \pm \mathrm{SD})$.

standard quantitative assay of antigen uptake by DC [9]. The results showed that the uptake of tumor protein by DC was significantly enhanced when tumor protein was conjugated to CNT $(P<0.05)$. The enhancements were $84 \%$ and $95 \%$ for TumorP concentration of $1 \mu \mathrm{g} / \mathrm{ml}$ and
$0.1 \mu \mathrm{g} / \mathrm{ml}$, respectively (Figure $1 \mathrm{C}$ and $1 \mathrm{D}$ ). Confocal microscopy indicated that the FITC-labeled protein was indeed taken into DC (Supplementary information, Figure S2). These results suggest that CNT might carry more tumor protein into DC and presumably would allow 
more tumor antigens to be intracellularly processed. The effects of CNT or CNT-TumorP on DC maturation were also evaluated. CNT or CNT-TumorP did not show a significant influence on the DC maturation process (Supplementary information, Figure S3)

Although CNT-TumorP increased the uptake of tumor antigen by DC, it was unknown whether the function of $\mathrm{DC}$ for inducing anticancer reaction was also affected. To study the issue, two groups of DC were first incubated with either TumorP or CNT-TumorP for 2 days, mixed with fresh autologous lymphocytes (effector cells) at the ratio of 1:10, and co-incubated with the MCF7 tumor cells (target cells) for 3 more days. The anticancer cytotoxiciy of the lymphocytes was then evaluated using a standard MTS cytotoxicity assay [10]. As shown in Figure 1E, the DC pretreated with CNTTumorP induced higher anti-tumor cytotoxicity $(P<$ $0.05)$ at most E:T ratios, suggesting that CNT-TumorP enhanced the function of DC to induce anticancer response in lymphocytes. This finding was consistent with the results shown in Figure 1D, where CNTTumorP increased the uptake of tumor protein by DC and presumably allowed more antigens to be processed. The above anticancer experiments involved a large number of DC that had been pre-expanded with in vitro culture. It would be interesting to explore whether CNT-TumorP could directly enhance the immune reaction of freshly obtained peripheral blood mononuclear cells (PBMC), which contained a more natural ratio of DC as in blood. To explore this issue, human PBMC were pretreated with CNT-TumorP and then evaluated for anticancer cytotoxicity. As shown in Figure 1F, the PBMC pretreated by CNT-TumorP consistently generated higher anti-tumor cytotoxicity compared to the other treatment groups $(P<0.05)$, suggesting that CNT-TumorP could indeed directly induce a more potent anticancer response in PBMC. It is important to investigate whether a direct CNT toxicity on the MCF7 cells was responsible for the higher tumor inhibition observed in Figure 1F. MCF7 cells were incubated for $72 \mathrm{~h}$ in either normal medium or media containing CNT of various concentrations. The viability of the MCF7 cells was then evaluated by standard MTS assay. The data showed no significant difference in the number of live cells among the groups (Figure $1 \mathrm{G}$ ), suggesting that the direct cytotoxicity of CNT was not the major mechanism of the enhanced anticancer cytotoxicity. To evaluate the specificity of the anticancer cytotoxicity induced by CNT-TumorP, human DC were pretreated with the MCF7 lysate protein conjugated to CNT (CNT-TumorP), and then co-incubated with autologous lymphocytes and various target cells including MCF7, HepG2 and CCC-ESF-1.
The results showed that DC-induced cytotoxicity was mainly against the MCF7 cells, but not the HepG2 or CCC-ESF-1 cells (Supplementary information, Figure S4), suggesting that anticancer cytotoxicity induced by CNT-TumorP was relatively specific against the tumor from which the tumor protein was extracted.

Dendritic cells are the most potent antigen presenting cells that can initiate primary immune responses when pulsed with antigens. An important type of modern anticancer immunotherapy is DC-based therapy, which is being actively evaluated in clinical trials and has generated promising results $[4,6]$. A primary concern of current DC therapy is to develop new technologies that can further enhance the efficacy of the available tumor antigens for DC stimulation. The aim of this study was to investigate whether CNT conjugated to tumor protein would influence the function of DC for processing tumor antigens. The results showed that CNT-TumorP could enhance the uptake of tumor antigen by human DC and the cytotoxicity induced by DC in vitro. It should be noted that the mechanism of CNT-facilitated uptake of tumor antigen by DC is not entirely clear and that further investigations are still warranted. Nevertheless, the results of the current study show that the function of DC can be further enhanced if tumor protein conjugated to CNT is used for DC simulation, suggesting that CNTbased nanotechnology may have potential applications in development of potent DC-based immunotherapy. Tumor lyaste, as a tumor antigen readily obtainable for immunotherapies, has generated promising results in some of the clinical trials $[4,6]$. The results of current study suggest that CNT may also prospectively be utilized to improve the efficacy of immunotherapies based on tumor lysates.

In summary, this study revealed that CNT-TumorP could enhance the uptake of tumor antigen by human DC and the capability of DC to induce anticancer response in vitro. The results suggest that CNT-based nanotechnology may have a prospective role in the development of more efficacious DC-based anticancer immunotherapy. Future studies may help to unveil the mechanism of CNT as a carrier for intracellular delivery of tumor antigen and to optimize the anticancer immune reaction facilitated by CNT.

\section{Acknowledgments}

We acknowledge the funding support from the China Medical Board and the Chinese Ministry of Science and Technology (2006CB933204, 90306004, 90406024).

Zhao Sun ${ }^{1, *}$, Wei Wang ${ }^{2, *}$, Jie Meng ${ }^{2}$, Shuchang Chen ${ }^{1}$, Haiyan $\mathrm{Xu}^{2}$, Xian-Da Yang ${ }^{2}$ 
${ }^{I}$ Department of Oncology, Peking Union Medical College Hospital, Beijing 100730, China; ${ }^{2}$ Institute of Basic Medical Sciences, Peking Union Medical College \& Chinese Academy of Medical Sciences, Beijing 100730, China

*These two authors contributed equally to this work. Correspondence: Xian-Da Yang ${ }^{\mathrm{a}}$, Haiyan $\mathrm{Xu}^{\mathrm{b}}$

${ }^{\mathrm{a}}$ Tel/Fax: +86-10-65296488

E-mail: ayangmd@gmail.com

${ }^{\text {b}}$ Tel/Fax: +86-10-65296437

E-mail: xuhy@pumc.edu.cn

\section{References}

1 Vermorken JB, Claessen AM, van Tinteren H, et al. Active specific immunotherapy for stage II and stage III human colon cancer: a randomised trial. Lancet 1999; 353:345-350.

2 Takayama T, Sekine T, Makuuchi M, et al. Adoptive immunotherapy to lower postsurgical recurrence rates of hepatocellular carcinoma: a randomised trial. Lancet 2000; 356:802-807.

3 Berd D, Sato T, Maguire HC Jr, et al. Immunopharmacologic analysis of an autologous, hapten-modified human melanoma vaccine. J Clin Oncol 2004; 22:403-415.
4 Yu JS, Liu G, Ying H, et al. Vaccination with tumor lysatepulsed dendritic cells elicits antigen-specific, cytotoxic T-cells in patients with malignant glioma. Cancer Res 2004; 64:49734979.

5 Rosenberg SA Progress in human tumour immunology and immunotherapy. Nature 2001; 411:380-384.

6 Brossart P Dendritic cells in vaccination therapies of malignant diseases. Transfus Apher Sci 2002; 27:183-186.

7 Bianco A, Kostarelos K, Partidos CD, et al. Biomedical applications of functionalised carbon nanotubes. Chem Commun (Camb) 2005; 5:571-577.

8 Meng J, Meng J, Duan J, et al. Carbon nanotubes conjugated to tumor lysate protein enhance the efficacy of an antitumor immunotherapy. Small 2008; 4:1364-1370.

9 Jarnjak-Jankovic S, Hammerstad H, Saeboe-Larssen S, et al. A full scale comparative study of methods for generation of functional Dendritic cells for use as cancer vaccines. $B M C$ Cancer 2007; 7:119.

10 Haas C, Lulei M, Fournier P, et al. A tumor vaccine containing anti-CD3 and anti-CD28 bispecific antibodies triggers strong and durable antitumor activity in human lymphocytes. Int $J$ Cancer 2006; 118:658-667.

(Supplementary information is linked to the online version of the paper on the Cell Research website.) 